\title{
Análisis de un Programa Universitario de Formación de Postgrado en Endocrinología, luego de 24 años de su inicio
}

\author{
José M. López M, Carlos Fardella B. \\ Analysis of a university training \\ program in endocrinology
}

The Pontificia Universidad Católica de Chile has a two decades experience in training endocrinology specialists. This communication explains the operation of the training program and the results of a survey, answered by $90 \%$ of Endocrinologists that were trained at the center. This survey covers different aspects of the program such as the strengths and weaknesses of the academic teaching and the present position of the trainees. Questions about the working environment and the relationship with their teachers during the application of the program are also included. These results can be useful for other University centers that are planning a training program in endocrinology and for those physicians that are planning to become endocrinologists (Rev Méd Chile 2007; 135: 1202-8).

(Key words: Education, medical, graduate; Endocrinology; Questionnaires)

Recibido el 5 de diciembre, 2006. Aceptado el 4 de enero, 2007.

Departamento de Endocrinología, Facultad de Medicina, Pontificia Universidad Católica de Chile. Santiago de Chile.

$E^{n}$ 1984, con ocasión del 250 aniversario de la fundación de la Sociedad Chilena de Endocrinología y Metabolismo, se efectuó un estudio sobre algunos aspectos de la endocrinología de adultos en Chile ${ }^{1}$. Han pasado 22 años y son abundantes los cambios acaecidos en la endocrinología nacional y mundial. Un aspecto relevante, el de la formación de especialistas en endocrinología, es el tema de esta comunicación, a raíz de la

Correspondencia a: Drs. José Manuel López M y Carlos Fardella B. Departamento de Endocrinología, Facultad de Medicina. P. Universidad Católica de Chile. Lira 85, 5o piso, Santiago. Fono (562) 354-3095. Fax: (562) 638-5675. E mail: jmlopez@med.puc.cl - cfardella@med.puc.cl experiencia de más de dos décadas de funcionamiento de un programa en la Pontificia Universidad Católica de Chile.

En Chile, la formación de especialistas en endocrinología tiene 3 vías formales: 1) Cumpliendo un programa universitario acreditado, 2) Completando un período reglamentado de sadiestramiento en práctica», 3) Aprobando un programa en el extranjero. La Tabla 1 desglosa por quinquenios el número de especialistas certificados por la Corporación Nacional de Certificación de Especialidades Médicas (CONACEM) desde su fundación, en 1986. La vía de reconocimiento utilizada se señala en la Tabla 2.

A la fecha existen 4 programas universitarios de dos años de duración cada uno, acreditados por la Asociación de Facultades de Medicina de 
Tabla 1. Certificaciones de Especialistas en Endocrinología otorgadas por C ON ACEM (Chile)

\begin{tabular}{|lr|}
\hline Años & $\mathrm{n}^{\mathbf{0}}$ \\
\hline $1986-1990$ & 57 \\
$1991-1995$ & 6 \\
$1996-2000$ & 8 \\
$2001-2005$ & 20 \\
2006 & 1 \\
Total & 92 \\
\hline
\end{tabular}

\section{Tabla 2. Vías de certificación de la especialidad de Endocrinología en Chile}

\begin{tabular}{|lr|}
\hline Vía & $\mathrm{n}^{\underline{0}}$ \\
\hline Profesor universitario* & 8 \\
Programa universitario & 21 \\
Adiestramiento en práctica & 56 \\
Formación en el extranjero & 7 \\
Total & 92 \\
\hline
\end{tabular}

* Vía operante sólo hasta el año 2003.

Chile (ASOFAMECH), con un débito anual de 4,5 especialistas. Los sustentan la Universidad de Chile ( 2 cupos al año, en dos programas), la Pontificia Universidad Católica de Chile (2 cupos al año) y, recientemente, la Universidad de Valparaíso (1 ingreso cada dos años). Estas universidades otorgan el título de Especialista en Endocrinología; CONACEM $^{2}$ reconoce esa calidad de especialista a requerimiento del interesado, cuando la universidad correspondiente certifica que el candidato ha completado y aprobado satisfactoriamente el Programa, y éste, a su vez, está acreditado previamente por ASOFAMECH. Es requisito ineludible tener certificación previa de Especialista en Medicina Interna.

La vía del «adiestramiento en práctica» es para médicos internistas, certificados ante CONACEM como especialistas en Medicina Interna (la endocrinología es una especialidad derivada de esa disciplina) y que hayan trabajado, insertos en un grupo institucional de especialistas reconocidos, por un período mínimo de 5 años, en jornada de 22 horas semanales al menos, y con una relación contractual con la institución que sustenta ese Servicio. Para ser aceptado como postulante el candidato debe demostrar continuidad en el ejercicio de la especialidad hasta la fecha de su solicitud. Estos antecedentes son evaluados por el Comité de Certificación de la especialidad, $\mathrm{y}$, de ser adecuados, éste eleva la solicitud al Directorio Nacional de CONACEM; cuando este organismo los aprueba, fija fecha para el examen escrito correspondiente. El último requisito, habiendo cumplido satisfactoriamente los anteriores, es la aprobación del examen práctico en un Departamento o Unidad de Endocrinología bajo la responsabilidad directa de un Profesor Titular.

La vía de formación en el extranjero es aceptada por CONACEM si el programa cumplido acredita que sus contenidos son homólogos a los de los programas chilenos reconocidos por ASOFAMECH. Estos candidatos deben aprobar los exámenes escrito y práctico señalados precedentemente.

El primer hecho que resalta es el reducido número de programas acreditados por ASOFAME$\mathrm{CH}$ para formar endocrinólogos. Esta era ya una queja en el análisis de la endocrinología nacional de hace 20 años y se pedían acciones en este sentido; sin embargo, los avances han sido mínimos. Formar en todo el país nueve especialistas cada dos años es una cifra que está lejos de dar satisfacción al déficit acumulado y menos a las demandas futuras crecientes del país. Ello incluso se agrava al considerar que este esfuerzo involucra algunos médicos extranjeros que regresan a su país de origen, magnificando el déficit. Así, de las 13 regiones administrativas de Chile, 5 no cuentan con ningún endocrinólogo certificado; además, su distribución es irregular, con 4 regiones que sólo tienen 1 ó 2 especialistas. Estas cifras, puestas de otro modo, traducen que al menos 15 ciudades de 80.000 habitantes o más de nuestro país no cuentan con al menos un endocrinólogo. Las restantes ciudades, a su vez, están lejos de cubrir adecuadamente sus necesidades.

En 1987 sólo 11\% de los médicos y socios de la Sociedad Chilena de Endocrinología y Metabolismo (SOCHEM) encuestados ${ }^{1}$, reconocía haber adquirido la calidad de especialista endocrinólogo a través de un programa formal. La cifra actual no es muy distinta. Se necesita con urgencia de una 
política estatal, desarrollada a través de las universidades y sus programas acreditados para dar una solución a 10 años plazo a este candente problema. Un antecedente al respecto ha sido el exitoso programa de formación de especialistas en Anatomía Patológica efectuado por un centro formador universitario, con el apoyo del Ministerio de Salud a partir de 1975, lo que cambió el panorama claramente deficitario de esa especialidad en poco más de 10 años $^{3}$. Lamentablemente, hasta el momento no parece ser ésta la orientación de la autoridad sanitaria. La SOCHED, consciente del problema realiza un notable esfuerzo financiando un cupo de un Programa de Formación.

En forma supletoria, dada la insuficiencia anterior, se ha aceptado la formación a través de la práctica supervisada por al menos 5 años. Sin embargo, esta vía tiene serias dificultades, las mayores de las cuales son: a) el restringido número de grupos de especialistas asentados institucionalmente, capaces de dar sustento a programas de este tipo; b) mayoritariamente estas unidades o grupos están localizados en Santiago, dejando sin opción a muchos interesados de provincias; c) la dificultad de los candidatos de disponer al menos de media jornada para su formación; d) esta vía, que sólo requiere 22 horas semanales, está orientada a desarrollar contenidos netamente asistenciales. Esto último dificulta alcanzar una formación sistematizada y es la razón que explica las diferencias observables entre los especialistas que han conseguido su condición de tales según las dos vías analizadas.

La formación de médicos nacionales en el extranjero es una posibilidad, pero tiene escasa importancia numérica atendida su bajo número. La percepción actual es de dificultad creciente para conseguir cupos de formación clínica en los países desarrollados receptores de becarios. La mayoría de las becas de perfeccionamiento disponibles en áreas de la endocrinología, tienen un fuerte componente de investigación, lo que no llena la necesidad que la formación inicial requiere de programas de enseñanza clínica sistematizada y completa. Se suma a lo anterior el riesgo de que aquellos médicos que acceden a esta formación en el extranjero pueden no regresar a Chile, invalidando el esfuerzo.

El objetivo de la presente comunicación, es realizar una evaluación retrospectiva del Programa de Especialización en Endocrinología de la Pontificia Universidad Católica de Chile, desde sus inicios, hace 24 años, a la actualidad. En ella se evalúa, a través de una encuesta realizada a la casi totalidad de los médicos que lograron a través de él su formación académica, las fortalezas y debilidades que ésta presentó, así como la posición que ellos ocupan en la actualidad. Además, se incluyen preguntas acerca del entorno laboral y la relación con sus docentes en el desarrollo del programa. Al final, los autores, que han sido o son los profesores responsables del programa, comentan los hallazgos y plantean posibles soluciones. Creemos que esta presentación puede ser de utilidad para grupos universitarios que consideren desarrollar un Programa de Formación en Endocrinología y, por la información que aporta, también para futuros aspirantes a endocrinólogos.

El programa de la Pontificia Universidad Católica nació en 1982 con un enfoque eminentemente clínico y un diseño netamente tutorial. Desde su inicio ha exigido a sus alumnos dedicación exclusiva y jornada completa de trabajo, por lo que reciben un estipendio mensual. Transcurridos 24 años, han egresado 22 médicos del Programa, teniendo actualmente un débito de 2 especialistas por año. Del total de egresados 50\% son mujeres, y 13\% extranjeros. Las Escuelas de Medicina donde los candidatos obtuvieron su título de médico son variadas (Tabla 3). Los egresados del Programa de la Pontificia Universidad Católica de Chile representan $24 \%$ de los médicos especialistas reconocidos por CONACEM. Ellos trabajan en Santiago (55\%), provincias (32\%), o en el extranjero (13\%). El 20\% del total está ligado a alguna de cuatro

\section{Tabla 3. U niversidad que otorgó el T ítulo de M édico Cirujano a los alumnos del Programa de Formación de Especialistas en Endocrinología}

\begin{tabular}{|lcc|}
\hline Universidad & $\mathrm{n}$ & $(\%)$ \\
\hline Pontificia Universidad Católica & 8 & $(36)$ \\
Universidad de Chile & 5 & $(23)$ \\
Universidad de Concepción & 4 & $(18)$ \\
Universidad extranjera & 3 & $(14)$ \\
Universidad de La Frontera & 1 & $(4,5)$ \\
Universidad de Valparaíso & 1 & $(4,5)$ \\
\hline
\end{tabular}


Facultades de Medicina chilenas y, de ellos, la mitad pertenece al Departamento de Endocrinología de la Pontificia Universidad Católica de Chile.

El Programa tiene como objetivos capacitar a médicos internistas como especialistas en endocrinología de modo que ellos: 1) Puedan atender y resolver cualquier problema endocrinológico clínico; 2) Utilicen en forma eficiente los recursos conducentes a satisfacer el punto precedente; 3) Sean capaces de buscar, captar, interiorizarse e incorporar la nueva información generada por el avance de la disciplina; 4) Desarrollen el criterio científico que les facilite entender, plantear, discutir y ejecutar proyectos de investigación de la especialidad, y 5) Propendan a integrar sus conocimientos endocrinológicos con los de Medicina Interna y de Medicina General, sin perder de vista la concepción del hombre desde una perspectiva antropológica humanista y cristiana.

La atención ambulatoria representa 25\% del tiempo del Programa. El modelo docente pretende la formación del alumno tanto teórica como práctica. En lo teórico, se enfatizan los contenidos bioquímicos, fisiológicos y de biología molecular relacionados a la endocrinología. También considera las bases para la utilización y comprensión adecuada del laboratorio clínico, radiológico y radionuclear para el diagnóstico y tratamiento de las enfermedades endocrinológicas.

La mayor parte del Programa (aproximadamente 2/3) está dirigida al estudio clínico de las diferentes enfermedades endocrinológicas. En esta fase del Programa se enfatiza la expedición clínica de los becarios y con ello la capacidad de evaluar y tratar las diversas patologías de la especialidad. El Programa también capacita a los becarios en estudios básicos moleculares. Para lograr esta meta los alumnos realizan una estadía en laboratorio de biología molecular, durante 3 a 6 meses, lo cual les permite comprender los mecanismos de esta disciplina y ejecutar personalmente sus técnicas básicas, generalmente realizando un proyecto de investigación. Para esto último deben aprender el diseño, consentimiento informado, conducción, interpretación, análisis estadístico, presentación y publicación de los datos. Además, adquirir experiencia en la búsqueda, análisis y juicio crítico respecto de la literatura.

Para alcanzar los objetivos señalados el Programa cuenta con 10 profesores (4 Titulares, 3
Adjuntos, 2 Auxiliares y un Instructor). Las actividades en las que el alumno tiene responsabilidades definidas y por las cuales es evaluado son reuniones (clínica, médico quirúrgica, bibliográfica, histopatológica y de investigación), cursos, actividad asistencial y pruebas específicas. Entre los cursos formales destacan 1) Exploración neurorradiológica en patología selar y paraselar (12 sesiones); 2) Interpretación de mamografía y ecografía mamaria (12 sesiones); 3) Ecografía cervical diagnóstica y de apoyo a la punción de nódulos tiroideos (todo el período de formación); 5) Laboratorio bioquímico (4 sesiones); 6) Curso sobre Metodología Estadística en Investigación Clínica (10 sesiones); 7) La Escuela de Medicina ofrece también 12 cursos optativos sobre variados tópicos.

La actividad asistencial se desarrolla en función de: 1) Pacientes hospitalizados, privados 0 institucionales, en el Hospital Clínico y Hospital de la Asistencia Pública, según convenio vigente. Esta atención tiene una orientación netamente docente, con un profesor a cargo; 2) Atención ambulatoria supervisada, bisemanal, en el Consultorio externo de la especialidad. Este centro recibe anualmente un promedio de 6.000 pacientes seleccionados de la especialidad (no incluye diabetes mellitus); 3) Atención ambulatoria de endocrinología ginecológica con una frecuencia semanal durante dos meses.

En relación a la evaluación, los alumnos deben rendir una prueba de conocimientos al inicio del Programa, que permite conocer sus condiciones de ingreso, y de este modo, diseñar la estrategia y los énfasis en el aprendizaje. Al término del primer año se efectúa una prueba escrita que abarca toda la disciplina, con una distribución de preguntas de acuerdo a la prevalencia de las patologías. La estadía en Biología Molecular aporta una calificación independiente. A las notas anteriores se agregan las de concepto que cada docente tiene que proponer en razón de sus turnos mensuales de asistencia hospitalaria docente. A su vez, las presentaciones semanales en las reuniones del Departamento dan otra perspectiva de valoración. De la consideración de todo lo anterior el alumno obtiene una nota oficial anual, y luego de dos años, de presentación para el examen oral final. Este examen es rendido ante al menos tres profesores del Departamento de Endo- 
crinología y uno de la Dirección de Postgrado; el candidato es evaluado como Aprobado con Distinción, Aprobado o Rechazado.

En marzo de 2006 se aplicó una encuesta escrita a todos los egresados del Programa, la cual consideraba 4 aspectos generales: a) Resultados y repercusiones en la vida profesional derivadas del hecho de haber cursado el Programa; b) Fortalezas y deficiencias del Programa respecto de sus contenidos, técnica docente, medios para facilitar el aprendizaje y dedicación y calidad de los docentes; c) Formación en el método científico y la investigación de la especialidad; d) Opinión sobre la estructura de las unidades temáticas del Programa. Fue contestada usando una escala de 4 puntos o una nota entre 1 y 7.

La encuesta, escrita, fue respondida por $90 \%$ de los egresados. Las respuestas generales y sobre docencia se presentan en la Tabla 4.

En relación a los aspectos generales la totalidad de las respuestas consideró que los 2 años de programa llevan a un buen desempeño futuro de la especialidad, y permiten adquirir el método científico. La docencia también fue evaluada en cuanto a los docentes mismos y los medios siendo globalmente muy satisfactoria (Tabla 4).

Hubo consenso en que es muy útil para el control del programa y el conocimiento objetivo del desempeño de los alumnos tener evaluaciones formales escritas cada 6 meses $(65 \%$ de las respuestas) o anuales (30\%). La evaluación de los contenidos del programa fue muy positiva, con especial buena calificación en las áreas de tiroides, metabolismo óseo, suprarrenal y biología molecular (nota superior a 6,4). Sólo lo concerniente a gónada masculina, nota 5,1 y diabetes mellitus 5,2, lo cual se refrenda al ser señaladas también en otra pregunta como las únicas áreas con algún grado de limitación (35\% y 25\% de las respuestas, respectivamente).

Especial aceptación tuvieron los cursos formales sobre exploración neurorradiológica y otros, con notas superiores a 6,2. Sólo el de ecografía cervical tuvo una nota promedio de 5,9.

Un hecho llamativo al comparar la encuesta hecha en 1987 con los datos actuales es el paulatino cambio de especialistas hombres a mujeres. En aquella encuesta, las endocrinólogas representaban $20 \%$ del universo ${ }^{1}$, cifra que incluso incluía las endocrinólogas pediatras. El dato actual es que $50 \%$ de los médicos que hicieron el Programa son mujeres. Aún más y en el mismo sentido, la proporción de médicos hombres aceptados en nuestro Programa en la primera y última mitad de su existencia varió de $64 \%$ a $36 \%$ actual.

Los cupos del Programa para postulantes extranjeros han sido moderados (18\%); la mayoría de ellos han sido destinados a extranjeros cuando los candidatos nacionales no los han ocupado por distintas razones. Una de ellas, e importante, es la falencia de recursos para otorgar un estipendio por la Universidad a candidatos nacionales para que puedan cumplir con el requisito substancial del Programa que es la dedicación exclusiva. En

Tabla 4. Encuesta contestada por los egresados del Programa de Formación de Endocrinólogos PU C

I.- Aspectos generales. El Programa de formación, te permitió:

1) ¿Un buen desempeño como especialista?

2) ¿Aprender el método científico?

3) ¿Su duración de 2 años la consideras suficiente?

$\begin{array}{rrrr}\text { A } & \text { B } & \text { C } & \text { D } \\ 84 \% & 16 \% & & \\ 70 \% & 25 \% & 5 \% & \\ 75 \% & 10 \% & 10 \% & 5 \%\end{array}$

II.- Docencia. Los docentes del Programa:

1) ¿Fueron dedicados en su tarea y te apoyaron?

2) ¿Crearon un ambiente propicio a la enseñanza?

$60 \% \quad 40 \%$

3) ¿Dispusiste de los medios para tu formación?

$45 \% \quad 45 \% \quad 10 \%$

$70 \% \quad 30 \%$

( $\mathrm{A}=$ Sí, totalmente de acuerdo; $\mathrm{B}=\mathrm{Sí}$, de acuerdo; $\mathrm{C}=$ =Parcialmente en desacuerdo y $\mathrm{D}=$ =Mayormente en desacuerdo). 
los candidatos extranjeros, aunque exigida, la formación previa en Medicina Interna ha sido muy disímil lo que crea una situación de desbalance con los nacionales y una dificultad para lograr su nivelación; este hecho pone de manifiesto la importancia de que endocrinología siga siendo una especialidad derivada de la Medicina Interna $y$, en consecuencia, se exija formación previa en esta última disciplina.

El Programa de Endocrinología que analizamos ha sido exitoso en opinión de sus alumnos. Quizás los elementos que han estado presentes y deban considerarse como fortalezas son: 1) La dedicación exclusiva del alumno al programa; 2) La selección cuidadosa de los candidatos, que además de sus antecedentes curriculares, incluye entrevistas dedicadas a aquilatar su interés vocacional y su nivel cultural y evaluación sicológica profesional; 3) El seguimiento y evaluación ajustada del Programa y sus logros, lo que permite introducir modificaciones y puestas al día, 4) Contar con un número suficiente de docentes experimentados que otorga una efectiva asistencia docente y al alumno poder contrastar opiniones y modos de actuar; 5) El desarrollo y vivencia del método científico aplicado a la investigación con participación en proyectos de investigación y publicaciones derivadas; esta faceta entusiasma a los alumnos; 6) Contar con un centro de afluencia de pacientes de la especialidad que, por su diversidad, da respaldo real a la patología que considera el programa; 7) La integración efectiva con la endocrinología pediátrica. El beneficio de este trabajo mancomunado es especialmente notorio en la patología puberal o en el seguimiento de enfermedades de la infancia.

Así como hay fortalezas, existen algunos puntos que deberán ser mejorados. Uno de ellos es el lugar de la Diabetología en la formación de un especialista en Endocrinología. La experiencia en Chile y otros países es bivalente, es decir algunos consideran la Diabetología aparte y otros la incluyen dentro de la Endocrinología. De hecho, los cultores de estas disciplinas agrupados por sus intereses dieron origen a la Sociedad Chilena de Diabetes y Nutrición y a la Sociedad Chilena de Endocrinología y Metabolismo, respectivamente. Sólo en 1978 se fusionaron ambas entidades, pero mantienen un cierto corte diferencial en sus actividades académicas. Si se mira en términos de estructuración de los principales Servicios se da el caso de hospitales con separación de ellos (ej. San Juan de Dios, Salvador, Clínico Universidad Católica) y sin ella (Clínico José Joaquín Aguirre). Por último, los programas de formación están diseñados fundamentalmente en base a la disciplina endocrinológica; de hecho, CONACEM recientemente reconoció la especialidad de Diabetología como una entidad independiente. Las revistas especializadas no hacen sino refrendar esta división en la mayoría de los casos. Una teórica conjunción de ambas disciplinas en un mismo programa tiene el inconveniente de alargar la formación del especialista endocrinólogo. Por otro lado, la no profundización en diabetes es vista por los alumnos como una falencia, atendida la necesidad de tener que trabajar en centros que no consideran la división, que es lo que habitualmente sucede en provincias.

Otra área detectada con falencias fue la de ecotomografía cervical, situación superada desde 2006 al incorporar en forma permanente la pasada de los becarios por nuestro Centro de Tiroides, bajo la tuición de un médico radiólogo experto.

En relación a las perspectivas y futuro del programa se considera relevante el desarrollo de los siguientes puntos: 1) Expandir el cupo de alumnos. Creemos que existe la infraestructura, la organización y los docentes para permitir un egreso de hasta 4 becarios anuales. La limitante para cumplir con el requisito ineludible de dedicación exclusiva al programa es el financiamiento; será necesario sensibilizar a las autoridades nacionales (incluyendo probablemente cláusulas de destinación) y a otras entidades interesadas en el desarrollo de la especialidad. Sólo un aumento substancial en el ingreso lograría ir solucionando el déficit ya comentado. 2) Reforzar las áreas temáticas más débiles, e incorporar otras nuevas de relevancia. 3) Diseñar pasadas cortas y específicas en servicios ajenos a nuestra Universidad, especialmente en el extranjero. Un par de experiencias iniciales al respecto han sido muy estimulantes. 4) Desarrollar el intercambio docente con instituciones extranjeras, que permitan ampliar la perspectiva de los alumnos. 5) Postular a la acreditación del Programa por parte de los organismos evaluadores de EE.UU. de Norteamérica. 6) Ampliar la relación con los egresados, fundamentalmente por vía electrónica, proveyéndoles 
de educación continua a distancia. 7) Expandir la investigación a través de proyectos multicéntricos con otras universidades.

La tarea es amplia y dificultosa; sin embargo, la experiencia acumulada en más de dos décadas, la

\section{REFERENCIAS}

1. López JM, Rodríguez JA. Estudio sobre algunos aspectos de la Endocrinología en Chile. Rev Méd Chile 1987; 115: 976-82.

2. Corporación Nacional Autónoma de Certificación de Especialidades Médicas. (Conacem). http:// conacem.cl riqueza y atractivo de la especialidad, y la nítida necesidad de lograr solucionar la falencia de endocrinólogos en el país hace aumentar nuestro deseo de conseguirlo y destinar el esfuerzo consecuente.

3. BaRAhona R. Programa para formación de anátomo-patólogos según convenio entre la Posta Central de la Asistencia Pública y la Escuela de Medicina de la Universidad Católica. Archivos del Departamento de Anatomía Patológica de la Facultad de Medicina de la P. Universidad Católica de Chile. 1975. 